\title{
Testando teorias para o consumo agregado no Brasil
}

\author{
Testing theories of aggregate consumption in Brazil
}

Luckas Sabioni Lopes

Universidade Federal de Juiz de Fora

\begin{abstract}
In this paper, theories to describe aggregate consumption in Brazil are tested. Two datasets were utilized, one comprising yearly observations from 1947 to 2012, and another comprising quarterly observations, from the first quarter of 1991 to the second quarter of 2014. Our methodological approach was based on the use of BDS statistics to determine which theoretical and/or empirical model properly fits the features of time series. According to our results, the random-walk Permanent Income Hypothesis was not supported by the data, due to excess of sensitivity which can, to some extent, be explained by credit constraints. In this sense, a standard version of the consumption function with excess of sensitivity was estimated by assuming the error correction model. Once we accounted for (possible) multiple structural breaks in the latter consumption function, we cannot reject the null of IID residuals. Hence, we conclude that consumption in Brazil is better modeled as being sensitive to current income variations, which opens room for effective stabilization policies in the country.
\end{abstract}

\section{Keywords}

consumption; permanent income hypothesis; credit constraints; error correction model; BDS statistic.

JEL Codes D91; E21; E32.

\section{Resumo}

No presente artigo, foram testadas teorias para descrever o comportamento do consumo agregado brasileiro, considerando duas bases dados: uma anual, para o periodo de 1947 a 2012; e outra trimestral, abrangendo os anos de 1991 a 2014. A abordagem metodológica consistiu em utilizar a estatística BDS para validar a adequabilidade de modelos teóricos e empíricos às séries de tempo. Verificou-se que a versão passeio-aleatório da Hipótese da Renda Permanente não encontra suporte nos dados, em função de uma elevada sensibilidade do consumo à renda corrente. Característica que é apenas parcialmente explicada pelas restrições de acesso ao crédito. Em seguida, testou-se uma versão da função consumo com excesso de sensibilidade por meio do modelo de correção do erro. Após considerar a possibilidade de quebras estruturais múltiplas nesta última formulação, não foi possivel rejeitar a hipótese nula de resíduos IID. Conclui-se, portanto, que o consumo no Brasil é bem descrito como sendo sensivel aos desvios previstos da renda corrente, abrindo espaço para políticas anticíclicas que visem a suavizar as flutuações da atividade econômica no país.

\section{Palavras-chave}

consumo; hipótese da renda permanente; restrição ao crédito; modelo de correção do erro; estatística $B D S$.

Códigos JEL D91; E21; E32. 


\section{Introdução}

As teorias que procuram explicar o comportamento do consumo agregado ainda são objeto de amplo debate acadêmico. Tal fato é reflexo da importância desta variável para a composição do dispêndio total nas economias, para questões relacionadas ao crescimento econômico em longo prazo e aos ciclos de negócios. No Brasil, por exemplo, o consumo tem historicamente respondido por cerca de $65 \%$ do produto interno bruto (PIB), tendo crescido a uma taxa média de $2,5 \%$ ao ano desde a implantação do Plano Real, ${ }^{1}$ em 1994.

Ao longo dos últimos anos, duas correntes teóricas têm recebido bastante atenção no que concerne à explicação do comportamento do consumo: a que defende que (ou procura explicar por que) os agentes econômicos consomem uma fração constante da renda corrente; ${ }^{2}$ e aquela que afirma que os agentes consomem uma fração constante de sua renda permanente (a Hipótese da Renda Permanente, HRP).

Os desenvolvimentos iniciais da HRP são devidos a Friedman (1957). Contudo, foi com a publicação do trabalho de Hall (1978) que os estudos sobre a alocação intertemporal do consumo tomaram um novo rumo. Este autor argumentou que, sob certas condições, a hipótese de Friedman seria averiguada se a série de consumo se comportasse como um passeio aleatório, resultado que ficou conhecido na literatura como versão estocástica da HRP.

Todavia, mesmo Hall não apresentou evidências claras de que a série temporal do consumo dos Estados Unidos da América (EUA) seguisse tal processo. Posteriormente, Flavin (1981) e Hayashi (1982), entre outros, rejeitaram a HRP em sua versão estocástica naquele país, em favor de um excesso de sensibilidade do consumo à renda corrente.

De fato, estimativas de Campbell e Mankiw (1990) mostraram que cerca de $50 \%$ da renda agregada nos EUA pertence a indivíduos cujo consumo segue as variações previstas da renda corrente, resultado que tem sido largamente atribuído às restrições à liquidez. No Brasil, tal proporção tende a ser ainda maior. Reis et al. (1998), por exemplo, ao estudar séries

1 Consumo final, variação real. Dados do Instituto Brasileiro de Geografia e Estatística (IBGE, 2006). Séries disponíveis em http://www.ipeadata.gov.br/.

2 Veja-se Thomas (1989) para uma revisão histórica das primeiras formulações das funções consumo e dos métodos de estimação. Ademais, Attanasio e Weber (2010) fazem uma ampla revisão bibliográfica do estado da arte das teorias do consumo intertemporal, dos modelos econométricos e das evidências empíricas disponíveis para os países desenvolvidos. 
agregadas do consumo entre 1947 e 1994, verificaram que grande parcela da renda (entre 70 e $90 \%$ ) pertence a consumidores restritos no sentido de Campbell e Mankiw. Como alternativa às restrições à liquidez, os autores encontram evidências de que os consumidores brasileiros realizam poupança precaucional. Seus resultados mostram que períodos mais instáveis podem elevar a taxa de crescimento do consumo em até 1,6\% a.a.

Gomes (2004), objetivando estudar a HRP entre 1947 e 1999, contra as hipóteses de formação de hábitos e de restrições à liquidez, encontrou evidências de um componente cíclico no consumo, que foi estimado como um processo estocástico com raiz unitária e ciclos do tipo ARMA (1, 1), contrariando a hipótese de Hall (1978). Em seguida, o autor concluiu que esse componente cíclico se deve à alta sensibilidade do consumo à renda corrente, estimando a fração da renda dos consumidores restritos em cerca de $85 \%$.

Por sua vez, Gomes (2010) avaliou o comportamento do consumo agregado entre 1947 e 2005 sob a ótica de três teorias distintas, a saber: a hipótese do comportamento otimizador; a hipótese de consumidores restritos aos moldes de Campbell e Mankiw (1990); e, a hipótese de miopia de Shea (1995), que argumenta que se os consumidores enfrentam restrição ao crédito e não são míopes, eles deveriam responder assimetricamente aos períodos de crescimento e de queda da renda. As estimações do autor não deram suporte às hipóteses de otimização e de miopia, porém a sensibilidade do consumo à renda corrente foi alta, o que pode estar associado às restrições à liquidez.

Em Gomes e Paz (2004), analisou-se a HRP para um painel de países da América Latina entre 1951 e 2000, com a metodologia de Campbell e Mankiw (1990). A fração da renda dos consumidores restrita a consumir a renda corrente no Brasil foi cerca de $60 \%$, inferior à obtida pela maioria dos países da região. Por fim, Gomes e Paz (2010) analisaram, adicionalmente, a hipótese de miopia (Shea, 1995) para Brasil, Colômbia, Peru e Venezuela entre 1951 e 2000. No caso brasileiro, os autores encontram alguma evidência de restrições à liquidez, que foi enfraquecida, entretanto, pela não significância de alguns parâmetros do modelo econométrico utilizado.

Portanto, o resultado de maior destaque na literatura consultada para o caso brasileiro é a rejeição da hipótese de Hall (1978), dada a significativa parcela dos consumidores que segue a regra de bolso de consumir uma fração da renda corrente (conforme formulação de Campbell e Mankin, 1990). Este é o ponto de partida para a principal contribuição da 
presente pesquisa, qual seja: argumenta-se que é falho assumir a adequabilidade da função consumo com excesso de sensibilidade diretamente da rejeição da HRP de Friedman/Hall. A primeira teoria também precisa ser avaliada.

Nesse sentido, o principal objetivo deste artigo é testar, rigorosamente, qual hipótese é mais adequada para a explicação do consumo agregado no Brasil. Em outras palavras, objetiva-se verificar qual formulação, a HRP ou a formulação em que o consumo responde às mudanças previstas na renda corrente, é capaz de capturar todo o padrão de dependência temporal do agregado em tela. Para alcançar esse objetivo, faz-se um extensivo exercício econométrico inédito na literatura, com o uso do teste BDS, desenvolvido por Brock et al. (1996).

O teste BDS avalia a hipótese nula de que uma série temporal é gerada por um processo independente e identicamente distribuído (IID), contra as alternativas de dependência linear e/ou não linear. A lógica de sua utilização no presente contexto é a seguinte: se a série de consumo é gerada pelo processo descrito na HRP, então os resíduos de sua primeira diferença deveriam ser IID. Caso contrário, rejeita-se a formulação de Hall (1978). De maneira análoga, se a função com excesso de sensibilidade consegue explicar o comportamento do consumo agregado, então os resíduos oriundos dessa especificação deveriam ser IID. Assume-se o modelo de correção do erro (MCE, de Engle e Granger, 1987) como formulação para a função consumo que exibe alta dependência das variações previstas da renda. Ao passo que o MCE permite a modelagem de hábitos, ciclos e correção do erro na dinâmica do consumo, estende-se, assim, a análise de Gomes (2004).

Como segunda contribuição, testa-se especificamente se os desvios do comportamento otimizador, como previsto pela teoria de Hall (1978), podem ser explicados pelas restrições de crédito enfrentadas pelos consumidores, seguindo a abordagem proposta por Ludvigson (1999). Em caso afirmativo, é possível indicar em que medida as políticas que facilitam o acesso aos mercados financeiros e creditícios podem aproximar os consumidores de suas trajetórias ótimas, o que produz aumento de bem-estar.

O restante deste artigo é divido como a seguir. Na segunda seção, são revisadas a hipótese da renda permanente em sua versão estocástica e a formulação da função consumo dada pelo mecanismo de correção do erro. Na terceira seção, é explicado o teste BDS, sua aplicação como forma de avaliar a adequação dos modelos teóricos e discorre-se também sobre os 
dados e procedimentos utilizados. Na quarta seção, são reportados os resultados. Por fim, na quinta seção, são apresentadas as conclusões.

\section{Modelos teóricos e empíricos}

\subsection{Consumo ao longo do ciclo de vida: a hipótese de Hall}

A versão estocástica da hipótese da renda permanente de Hall (1978) tornou-se uma das abordagens mais utilizadas na literatura que estuda o comportamento do consumo agregado e um ponto de partida para diversas discussões teóricas e empíricas. Assim, expõe-se a seguir uma forma simplificada desse modelo queconduz aos resultados de interesse. Supõe-se um agente representativo que maximize sua utilidade ao longo do ciclo de vida, sujeito a uma restrição orçamentária intertemporal. Considera-se, ainda, que a função de utilidade assuma uma forma funcional quadrática. Tem-se, assim, o seguinte problema de otimização dinâmica condicionada (Romer, 2001),

$$
\begin{aligned}
& \text { Máx. } \mathrm{E}_{t}[U]=\mathrm{E}_{t}\left[\sum_{t=1}^{T}-a / 2\left(\bar{C}-C_{t}\right)^{2}\right] \\
& \text { s.a. } \sum_{\mathrm{t}=1}^{\mathrm{T}} C_{t} \leq A_{0}+\sum_{t=1}^{T} Y_{t} .
\end{aligned}
$$

Em que $\mathrm{E}_{t}[$.$] representa a esperança matemática de uma variável condicio-$ nada a toda informação disponível no período $t$, com $t=1, \ldots, \mathrm{T}$; $U$ é o nível de utilidade do consumo; $C_{t}$ e $Y_{t}$ são o consumo e a renda corrente, respectivamente; a e $\bar{C}$ são parâmetros positivos; e $A_{0}$ é o estoque inicial de riqueza. Nota-se que, por simplicidade de exposição, desconsideram-se as taxas de juros e de desconto intertemporal do consumo, hipótese relatada à frente. Em adição, a equação (2) diz que o somatório do consumo deve ser menor ou igual ao estoque inicial de riqueza $\left(A_{0}\right)$ mais a renda corrente e futura, de maneira que o indivíduo não possua dívidas ao fim do ciclo de vida. As condições de primeira ordem são facilmente obtidas, e com a obediência à (2) em sua igualdade, pode-se mostrar que:

$$
\begin{aligned}
& C_{1}=\frac{1}{T}\left(A_{0}+\sum_{t=1}^{T} \mathrm{E}_{1}\left[Y_{t}\right]\right), \\
& \mathrm{E}_{t}\left[C_{t+1}\right]=C_{t}, \text { ou, } C_{t+1}=C_{t}+\varepsilon_{t+1}
\end{aligned}
$$


Em que $\varepsilon_{t}$ denota um termo ruído branco. A equação (3) é a conclusão central de Friedman (1957). Ela mostra que o consumo corrente de um indivíduo, $C_{1}$, é uma média da expectativa de sua renda corrente e futura, adicionada de algum estoque inicial de riqueza, ou seja, a teoria da renda permanente. Por sua vez, a equação (4) é a principal contribuição teórica fornecida por Hall (1978), em que a série do consumo, ao longo da trajetória ótima, deveria se comportar como um passeio aleatório. ${ }^{3}$

No contexto de políticas macroeconômicas, a verificação HRP reforçaria a visão de que somente movimentos imprevistos nas políticas fiscais ou monetárias afetariam consumo e renda agregados. Adicionalmente, mesmo as políticas não antecipadas teriam seu efeito reduzido, pois o consumo corrente responderia pouco aos movimentos transitórios da renda, diminuindo a relevância dos efeitos multiplicadores.

Entretanto, como relatado na introdução, as evidências empíricas não têm sido favoráveis à verificação da HRP, o que levou ao desenvolvimento de vários estudos que indagam as razões do distanciamento da série de tempo de sua trajetória teoricamente ótima. Uma resposta para isso se deve às restrições à liquidez (Hall; Mishkin, 1982; Campbell; Mankiw, 1990).

Nesse sentido, Campbell e Mankiw (1990) propuseram o método a seguir para estimar a fração da renda dos consumidores que não seguem a HRP de Friedman/Hall (equações 5 e 6, abaixo). Tal especificação decorre diretamente da suposição de que existam dois grupos de consumidores na economia, um que se comporta como previsto pela teoria de Hall (1978) e outro que consome uma fração fixa da renda corrente $(\lambda)$.

$$
\begin{aligned}
& \Delta C_{t}=\lambda \Delta Y_{t}+(1-\lambda) \varepsilon_{t}, \text { ou } \\
& \Delta C_{t}=\lambda \Delta Y_{t}+v_{t} .
\end{aligned}
$$

A equação (6) pode ser utilizada para a estimação de $\lambda$, a fração da renda dos agentes que não seguem a HRP. No Brasil, seu valor tem sido estimado em 85\% ou mais (ver Reis et al., 1998; Gomes, 2004; 2010). Neste artigo, a Hipótese da Renda Permanente, equação (4), é testada utilizando-se a metodologia descrita na próxima seção. Para fins comparativos, são produzidas estimativas para a equação (6) segundo o método proposto por 3 Incluir um termo drift, ou considerar que o logaritmo do consumo em (4) não altera a ideia central de Hall (1978). 
Campbell e Mankiw (1990), que utiliza os mínimos quadrados ordinários de dois estágios.

Apesar da estimação de valores altos para $\lambda$ ter sido amplamente associada à presença de restrições à liquidez, ${ }^{4}$ há uma escassez na literatura econômica brasileira de estudos que avaliem especificamente essa hipótese. $O$ presente artigo pretende cobrir tal lacuna, empregando a abordagem de Ludvigson (1999). Este autor desenvolve um modelo com agentes racionais e forward looking, em que os limites de crédito ao consumidor (ou seja, a restrição à liquidez) variam com o tempo, estando associados à evolução da renda corrente. Argumenta-se que o acesso ao crédito está atrelado à capacidade de pagamento dos tomadores de empréstimos. Contudo, como há problemas de informação assimétrica, é comum que os emprestadores considerem a renda corrente como uma boa aproximação da saúde e da riqueza financeira dos consumidores.

Nesse contexto, o modelo de Ludvigson (1999) produz uma causação direta do crescimento previsto do crédito sobre a taxa de crescimento do consumo, do crescimento previsto da renda sobre o crescimento do crédito e do crescimento previsto da renda sobre a taxa de crescimento do consumo, conforme ilustrado em (6). Para verificar essas propriedades teóricas nas séries de tempo observadas, o autor desenvolve a seguinte equação de teste:

$$
\Delta C_{t}=\mu+\lambda \Delta Y_{t}+\theta r_{t}+\alpha \Delta D_{t}+\varepsilon_{t}
$$

em que, na equação (7) são adicionadas a taxa de juros real, $r$; a variação do volume de crédito ao consumidor, $\Delta D_{i}$; e, um termo de erro aleatório, $\varepsilon$. O sinal esperado dos parâmetros $\lambda, \theta$ e $\alpha$ é positivo. Como anteriormente, $\lambda$ representa a fração da renda dos consumidores que seguem a renda corrente; a não rejeição de $\theta=0$ fornece evidências adicionais contra a hipótese da renda permanente; e, a significância estatística de $\alpha$ atesta para a presença de restrições à liquidez impostas pelas limitações de acesso ao crédito. A estimação de (7) é realizada com o método de mínimos quadrados de dois estágios.

Após os testes descritos terem sido realizados, é pertinente verificar se a função consumo com excesso de sensibilidade à renda corrente é adequa-

4 Browning e Crossley (2001) listam outras possíveis razões para um $\lambda$ estatisticamente significativo, como a poupança pelo motivo precaução (Nagatani, 1972), a regra psicológica de Keynes (1936) e custos de autocontrole (Shefrin; Thaler, 1988; Thaler, 1990). 
da aos dados, capturando toda a dependência temporal do agregado em questão. A intuição dessa função é fornecida a seguir, na próxima subseção.

\subsection{Alta sensibilidade do consumo à renda corrente: o modelo de correção do erro}

Segundo Thomas (1989), funções na forma $C_{t}=f\left(Y_{t}\right)$ têm longa tradição na literatura econômica. Desde 1937, diversos autores vêm utilizando especificações dessa natureza para descrever as propriedades básicas das séries de tempo do consumo. Conforme descrito por Davidson et al. (1978), as principais características a serem capturadas pelos modelos são: a) o consumo responde às mudanças previstas na renda corrente disponível; e $b$ ) existe uma diferença entre as propensões marginais a consumir (PMgC) em curto e em longo prazo, com a última sendo substancialmente maior.

Davidson et al. (1978) apresentaram, ainda, evidências empíricas e teóricas de que modelos de correção do erro (MCE) seriam capazes de reproduzir os fatos "a" e "b". Mais tarde, a econometria dos modelos MCE foi aprofundada por Engle e Granger (1987), em uma obra seminal sobre cointegração. Basicamente, supõe-se que os agentes queiram manter uma relação razoavelmente estável entre a renda corrente e o consumo em longo prazo. Entretanto, a possibilidade de ocorrência de desvios nesse planejamento, por qualquer razão que seja, cria a necessidade de ajustamentos periódicos por parte dos consumidores. Matematicamente, o modelo estimado neste artigo apresenta a seguinte forma:

$$
\begin{aligned}
& C_{t}=a+b Y_{t}+e_{t} \\
& \Delta C_{t}=\beta_{0}+\beta_{1} \Delta Y_{t}+\beta_{2} \Delta Y_{t-1}+\beta_{3} \Delta C_{t-1}+\gamma e_{t-1}+u_{t} .
\end{aligned}
$$

A equação (8) se refere à clássica função consumo, $C_{t}=f\left(Y_{t}\right)$, com $a>$ 0 e $0<b \leq 1$. Se as séries temporais da renda, $Y$, e do consumo, $C$, são cointegradas, então (8) é a relação de equilíbrio de longo prazo planejada pelos consumidores. Com intuito de testar a hipótese de cointegração, utiliza-se o método proposto por Engle e Granger (1987), o qual verifica a estacionariedade dos resíduos de (8), i.e., $e_{t}$. Em seguida, na equação (9), 
também conhecida como mecanismo de correção do erro, são representadas as respostas do consumo aos desvios de curto prazo na renda corrente e defasada, $\Delta Y_{t}$, e $\Delta Y_{t-1}$, às variações defasadas do consumo, $\Delta C_{t-1}$, e aos desvios em torno da relação de longo prazo, $e_{t-1}$, corrigidos pelo coeficiente de ajustamento, $\gamma$.

As equações (8) e (9) encontram suporte empírico (Davidson et al., 1978) e têm forte apelo intuitivo. Nota-se, por exemplo, que as $\mathrm{PMgC}$ em longo e curto prazo são distintas, denotadas, nesta ordem, pelos parâmetros $b$ e $\beta_{1}$. Ademais, é esperado que o parâmetro $b$ seja estimado próximo da unidade. Tal fenômeno pode ser explicado pelas restrições à liquidez impostas pelo mercado de crédito, que forçam os indivíduos a consumir a totalidade da renda, haja vista a impossibilidade de suavizar o consumo (Hayashi, 1982; Deaton, 1989; Ludvigson, 1999, entre outros); como também pela maior preferência por ativos de baixa liquidez para formar poupança, implicando que "consumption tracks income" (Shefrin; Thaler, 1988, p. 629); ou mesmo pela regra psicológica de Keynes (1936). Os termos $\Delta Y_{t-1} \mathrm{e}$ $\Delta C_{t-1}$ introduzem maior riqueza dinâmica ao modelo estimado, possibilitando persistência temporal e formação de hábitos (como em Fuhrer, 2000; ou Weber, 2002). ${ }^{5}$ Por fim, os desvios defasados ao longo da trajetória de equilíbrio do consumo, et-1, são corrigidos período a período com a velocidade do ajuste medida por $\gamma$.

A questão central a ser investigada neste artigo é, portanto: qual modelo entre aqueles apresentados nas equações (4), (8) e (9) consegue responder por todo o padrão de dependência temporal na série do consumo agregado? A resposta a este tipo de indagação fornece subsídios essenciais à formulação de políticas, seja de caráter de curto prazo, relacionadas à mitigação dos ciclos econômicos, seja de caráter de longo prazo, ao proporcionar melhor entendimento da função consumo e, assim, da poupança na economia brasileira. Detalhes adicionais acerca da estimação do MCE,

5 Conforme Gomes (2004) explica, modelos teóricos com formação de hábitos foram estimulados pela persistência existente nas séries de consumo. Assim, uma vez que elas se distanciam da tendência de longo prazo, para cima ou para baixo, tendem a permanecer nesse estado por algum tempo, exibindo uma inércia associada com a formação de hábitos de consumo. A modificação da utilidade na equação (1) para a forma $U=\left(C_{t}, C_{t-1}\right)=-a / 2\left[\bar{C}-\left(C_{t}-h C_{t-1}\right)\right]^{2}$, em que $h$ é o parâmetro que representa a intensidade da formação de hábito, conduz a uma equação de teste similar a (9), com a exceção de que nela força-se o parâmetro $\gamma$ a zero. Isto é, (9) pode ser entendida como um processo temporal mais amplo, em que os agentes corrigem não somente os desvios do consumo de sua tendência, mas também os desvios ao longo da relação de longo prazo entre consumo e renda corrente. 
inclusive quanto à possibilidade de quebras estruturais, são fornecidos na seção de resultados.

\section{Método e base de dados}

\subsection{0 teste BDS e sua aplicação às teorias do consumo agregado}

O teste BDS, desenvolvido por Brock et al. (1996), mede o grau de dependência temporal em uma série. Sua hipótese nula de que os dados são um processo IID pode ser testada contra uma variedade de hipóteses alternativas, como a existência de dependência linear ou não linear. Dessa forma, o teste BDS vem sendo largamente aplicado ao teste de especificações econométricas. Algumas aplicações relacionadas ao presente artigo são devidas à Barnett e Serletis (2000), Chen e Yeh (2002) e Rahman e Saadi (2008), que verificam a hipótese de mercados eficientes de Fama (1970).

A estrutura do teste BDS se dá como a seguir. Escolhe-se uma distância máxima de comparação de pontos, $\in$, e define-se $p_{1}(\in)$ como a probabilidade da distância entre quaisquer pares de pontos ser menor ou igual a $\in$. Constroem-se, agora, conjuntos consecutivos de pares de observações, isto é, dados os pontos $\varepsilon_{s}$ e $\varepsilon_{t}$ de uma série temporal, faz-se o seguinte: $\left\{\left[\varepsilon_{s}, \varepsilon_{t}\right],\left[\varepsilon_{s+1}, \varepsilon_{t+1}\right],\left[\varepsilon_{s+2}, \varepsilon_{t+2}\right], \ldots,\left[\varepsilon_{s+m-1}, \varepsilon_{t+m-1}\right]\right\}$, em que $m$ é o comprimento do conjunto, também conhecido como dimensão de imersão.

Denota-se, então, a probabilidade conjunta de todos os pares de pontos pertencentes ao conjunto anterior satisfazerem a condição de $\in$ por $p_{m}(\in)$. Sob a suposição IID, essa probabilidade será simplesmente o produto das probabilidades individuais de cada par, ou seja,

$$
p_{m}(\in)=p_{1}^{m}(\in)
$$

No caso de dados amostrais, $p_{m}(\in)$ e $p_{1}(\in)$ devem ser estimadas e, por isso, a equação (10) não é verificada exatamente, mas sim com algum erro. Quanto maior este erro, menor a probabilidade de que ele tenha sido gerado aleatoriamente. Assim, a estatística BDS fornece uma base formal para o julgamento do tamanho desse erro.

Para estimar a probabilidade em uma dimensão em particular, coletam-se todos os possíveis grupos de uma dada duração (intervalo de tempo 
entre pontos) e observam-se quantos deles obedeceram à condição $\in$. A razão desses conjuntos pelo total de conjuntos possíveis de serem formados fornece a estimativa da probabilidade. Assim, com n pontos amostrais, a formulação matemática dessa probabilidade é:

$$
p_{m, n}(\in)=\frac{2}{(n-m+1)(n-m)} \sum_{s=1}^{n-m+1} \sum_{t=s+1}^{n-m+1} \prod_{j=0}^{m-1} I_{\epsilon}\left(\varepsilon_{s+j}, \varepsilon_{t+j}\right)
$$

em que $I_{\epsilon}$ é uma função indicador que assume o valor 1 se o módulo da distância entre dois pontos for menor ou igual $a \in$ e zero, caso contrário. A estatística $p_{m, n}$ é conhecida como integral de correlação. ${ }^{6}$

Desse modo, o teste de independência é construído da seguinte maneira:

$$
b_{m, n}(\in)=p_{m, n}(\in)-p_{1, n-m+1}(\in)^{m},
$$

em que, sob a suposição de independência, Brock et al. (1996) mostraram que $(\sqrt{n-m+1}) b_{m, n} / \sigma_{m, n}$ segue uma distribuição aproximadamente normal, com média zero e variância 1 (em que $\sigma_{m, n}$ representa o desvio padrão amostral da estatística de teste, $b_{m, n}$ ). Assim, o p-valor da hipótese nula pode ser calculado a partir das informações da distribuição normal padronizada. Entretanto, neste artigo também são analisados os p-valores gerados por simulações bootstrap, em função da curta duração das séries. A fórmula para o cálculo de $\sigma_{m, n}^{2}$ pode ser consultada no artigo de Brock et al. (1996, p. 205).

A estatística ilustrada pela equação (12) é utilizada para verificar a adequação dos modelos teóricos. A hipótese da renda permanente em sua versão estocástica implica que o consumo segue um processo passeio aleatório, sendo o teste BDS aplicado às primeiras diferenças das séries consideradas para o consumo. Ressalta-se que, como previsto pela HRP, o consumo segue um processo tal como $\left(C_{t}-C_{t-1}\right)=\varepsilon_{t}$. Assim, caso haja evidências contra a hipótese nula de erros IID na primeira diferença do consumo (ou do logaritmo desta variável), tal resultado indica a rejeição da HRP, pois implica que de alguma forma os desvios do consumo estão conectados no tempo. No caso do teste da versão MCE da função consumo, o teste BDS é aplicado sobre os resíduos da equação (9).

6 A notação padrão da integral de correlação é $c_{m, n}$, porém aqui se utiliza $p_{m, n}$ para evitar confusão com a notação do consumo agregado, $C_{t}$. 


\subsection{Base de dados}

As séries anuais utilizadas para a estimação de (4), (8) e (9) são as do consumo final das famílias e da renda nacional bruta, para todo o período de 1947 a 2012, totalizando 66 observações ${ }^{7}$. Os dados originais, disponibilizados pelo Instituto Brasileiro de Geografia e Estatística, estão medidos em milhões de reais correntes. Assim, utilizou-se o deflator implícito do PIB, base em 1980, para retirar os efeitos das variações de preços para, em seguida, aplicar a transformação logarítmica. A Figura 1 ilustra as séries em nível, nas partes $a$ e $b$, e em primeira diferença, nas partes $c$ e $d$.

Figura 1 Séries anuais - 1947/2012
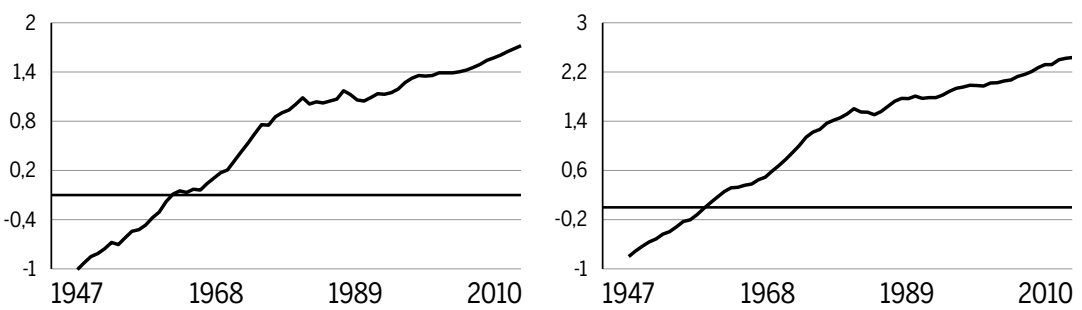

(a)

(b)
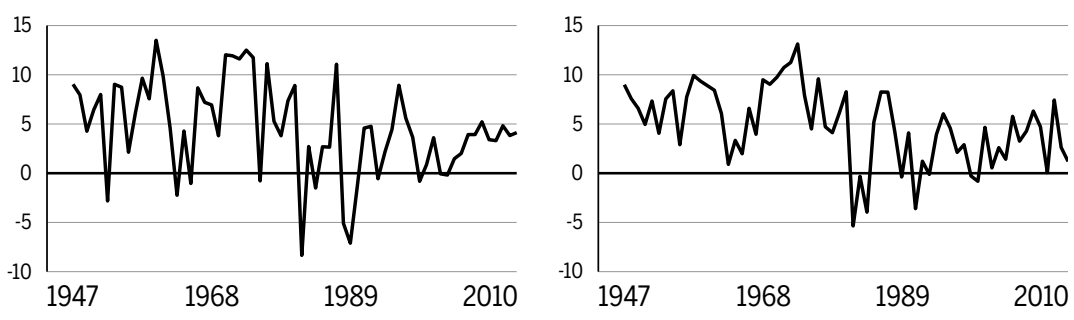

(c)

(d)

Fonte: Instituto Brasileiro de Geografia e Estatística e resultados da pesquisa.

(a) logaritmo do consumo das famílias; (b) logaritmo da renda; (c) primeira diferença do logaritmo do consumo; (d) primeira diferença do logaritmo da renda.

7 Com respeito à agregação das séries de consumo, Mankiw (1982) pondera que bens de consumo não duráveis e duráveis podem ter comportamento diferente. Argumenta-se que o consumo de bens duráveis segue um padrão cíclico, alinhado com as oscilações da atividade econômica, enquanto que o consumo de não duráveis segue um processo passeio aleatório. A esse respeito, Gomes, Issler e Salvato (2005) sugerem que no Brasil as séries de consumo podem ser consideradas conjuntamente, estratégia adotada no presente estudo. 
Ponderações acerca de possíveis quebras estruturais nas séries são feitas na seção de resultados. Especificamente, no caso da equação (4), em função da necessidade de uma série temporal relativamente longa para a implementação do teste BDS nos casos em que se avalia a HRP, as análises realizadas nas subamostras utilizam o índice encadeado trimestral do consumo final das famílias, série em valores reais, dessazonalizada e disponível para o período do primeiro trimestre de 1991 ao segundo trimestre de 2014, contando com 94 observações. A Figura 2 traz o consumo trimestral em logaritmos e sua primeira diferença, nas partes a e $b$, respectivamente. ${ }^{8}$

\section{Figura 2 Séries trimestrais do consumo - 1991/2014}
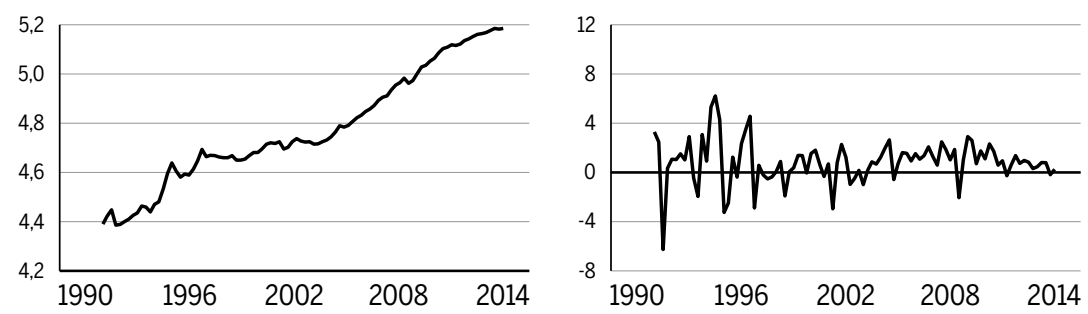

(a)

(b)

Fonte: Instituto Brasileiro de Geografia e Estatística e resultados da pesquisa.

(a) logaritmo do consumo das famílias; (b) primeiras diferenças do logaritmo do consumo.

A estimação da equação (7) foi realizada com dados trimestrais, variando do primeiro trimestre de 1991 ao último de 2012, em função da limitação imposta pela série do PIB real, disponibilizada por Bonelli e Rodrigues (2012). A série do consumo é a mesma apresentada na Figura (2). A taxa de juros real é medida ex post, por meio da subtração da inflação da taxa de juros nominal. A inflação é dada pela acumulação do Índice Geral de Preços, disponibilidade interna, mensal (IGP-DI). Por sua vez, para medir a taxa de juros, usa-se a acumulação da taxa básica mensal, a Selic. O crédito ao consumidor é medido pelo volume de crédito contraído por pessoas físicas. ${ }^{9}$ Quando necessário, as séries foram deflacionadas (IGP-DI) e

8 Série medida pelo Instituto Brasileiro de Geografia e Estatística, IBGE, e disponibilizada no endereço http://www.ipeadata.gov.br/.

9 A taxa Selic e o volume de crédito são mensurados pelo Banco Central do Brasil. A inflação medida pelo IGP-DI é calculada pela Fundação Getúlio Vargas. Esses dados estão publicados 
dessazonalizadas com o método X-12 aditivo. Ressalta-se, ainda, que em todas as estimações e testes realizados, as variáveis estão em logaritmos. A Figura 3 exibe os dados do PIB, da taxa de juros real, do volume de crédito e de suas variações percentuais.

Figura 3 Séries trimestrais do PIB, taxa de juros real e volume de crédito - 1991/2012
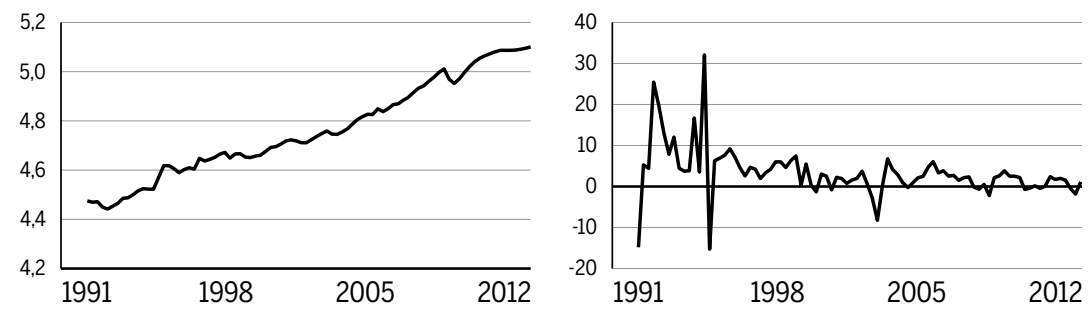

(a)

(b)
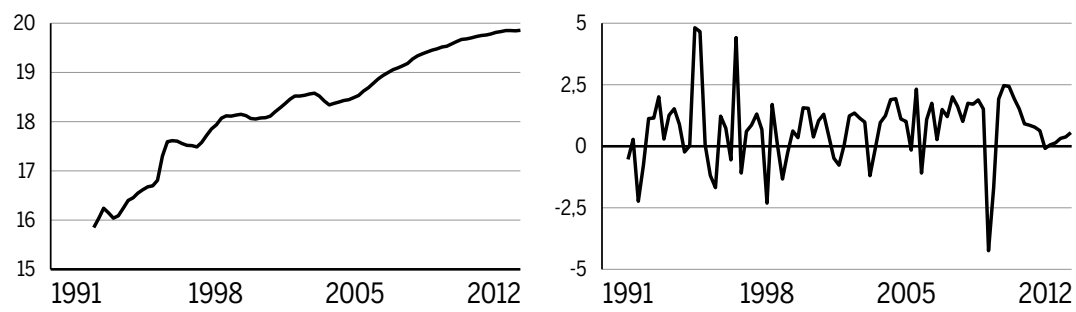

(c)

(d)

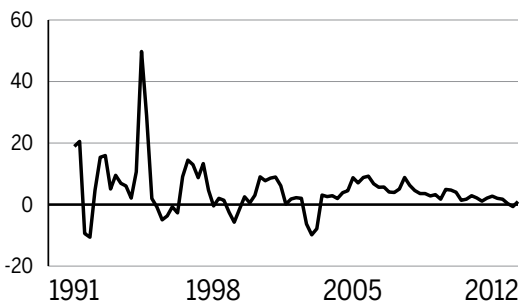

(e)

Fonte: Bonelli e Rodrigues (2012), Banco Central do Brasil, Fundação Getúlio Vargas e resultados do autor. (a) logaritmo do PIB real; (b) taxa de juros real; (c) volume de crédito; (d) primeiras diferenças do logaritmo do PIB; (e) primeira diferença do logaritmo do volume de crédito às pessoas físicas. 


\section{Resultados}

\subsection{Teste da versão estocástica hipótese da renda permanente}

Na primeira parte da seção de resultados, avalia-se a Hipótese da Renda Permanente na versão de Hall (1978) para o caso brasileiro. Com essa finalidade, a Tabela 1 traz as estatísticas BDS estimadas para a primeira diferença do log-consumo, considerando os dados anuais entre 1947 e 2012. Como é de praxe na literatura (veja-se Hsieh, 1989, por exemplo), os testes foram realizados para os casos em que a dimensão de imersão, $m$, varia de dois a seis, e o parâmetro $\in$ entre 0,5 e dois desvios padrão da série em questão, i.e., $\sigma_{c}$.

Tabela 1 Teste de independência dos resíduos da primeira diferença da série do log-consumo, dados anuais, 1947-2012

\begin{tabular}{|c|c|c|c|c|c|c|c|c|c|c|c|c|}
\hline \multirow{2}{*}{$m$} & \multicolumn{3}{|c|}{$\epsilon=0,5 \sigma_{c}$} & \multicolumn{3}{|c|}{$\epsilon=1 \sigma_{c}$} & \multicolumn{3}{|c|}{$\epsilon=1,5 \sigma_{c}$} & \multicolumn{3}{|c|}{$\epsilon=2 \sigma_{c}$} \\
\hline & $B D S$ & $P_{n}$ & $\boldsymbol{P}_{b}$ & $B D S$ & $\boldsymbol{P}_{n}$ & $P_{b}$ & $B D S$ & $P_{n}$ & $\boldsymbol{P}_{b}$ & $B D S$ & $P_{n}$ & $P_{b}$ \\
\hline 2 & 3,7 & 0,00 & 0,00 & 3,9 & 0,00 & 0,00 & 4,5 & 0,00 & 0,00 & 4,0 & 0,00 & 0,01 \\
\hline 3 & 5,0 & 0,00 & 0,00 & 4,4 & 0,00 & 0,00 & 4,5 & 0,00 & 0,00 & 4,4 & 0,00 & 0,00 \\
\hline 4 & 4,5 & 0,00 & 0,01 & 4,6 & 0,00 & 0,00 & 4,7 & 0,00 & 0,00 & 4,9 & 0,00 & 0,00 \\
\hline 5 & 4,1 & 0,00 & 0,03 & 4,8 & 0,00 & 0,00 & 4,8 & 0,00 & 0,00 & 4,9 & 0,00 & 0,00 \\
\hline 6 & 3,4 & 0,00 & 0,07 & 4,7 & 0,00 & 0,00 & 4,8 & 0,00 & 0,00 & 4,8 & 0,00 & 0,00 \\
\hline
\end{tabular}

Fonte: Resultados da pesquisa.

Nota: 66 observações; $m$ representa a dimensão de imersão; BDS, a estatística de teste padronizada; $\mathrm{P}_{\mathrm{n}}$, op-valor da distribuição normal; $\mathrm{P}_{\mathrm{b}}$, o $\mathrm{p}$-valor gerado por 10.000 simulações bootstrap; $e \sigma_{\mathrm{c}}$ refere-se ao desvio padrão estimado do consumo.

Os resultados expostos na Tabela 1 mostram que a estatística de teste para a verificação da HRP é bastante elevada. Na grande maioria dos casos, estima-se um valor superior a quatro para as estatísticas BDS, o que conduz à rejeição da hipótese nula de que os desvios do consumo agregado são IID com bastante segurança. Existem, portanto, dinâmicas temporais (lineares ou não lineares) passíveis de serem modeladas nas variações anuais do consumo agregado das famílias, isto é, há previsibilidade nas variações desta série. Assim, a Tabela 1 fornece evidências iniciais contrárias à hipótese da renda permanente para o caso brasileiro, resultado em consonância com o encontrado por Gomes (2004, 2010). 
O método descrito por Campbell e Mankiw (1990) para os dados anuais do logaritmo do consumo final das famílias e da renda, fornecendo uma estimativa para o parâmetro $\lambda$ da equação (6), é exibido na Tabela A.1 do anexo. Conforme sugerido pelos autores, é utilizado o método de mínimos quadrados ordinários e de dois estágios, com a lista de instrumentos ilustrada na segunda coluna. A última coluna da tabela apresenta a estatística calculada para o teste de endogeneidade de Durbin-Wu-Hausman (DWH), que tem como hipótese nula a exogeneidade do regressor $\Delta Y_{\mathrm{t}}^{10}$.

Como registrado pela Tabela A.1, as estimativas de $\lambda$ são, na metade das vezes, maiores que $90 \%$, indicando que parcela significativa da população brasileira se distancia da HRP. De maneira interessante, as estatísticas do teste DWH não permitem rejeitar a hipótese de que as variações contemporâneas da renda sejam exógenas. Neste caso, o valor de $\lambda$ pelo método de mínimos quadrados ordinários, igual a 0,94 , é obtido de maneira eficiente e consistente. Vale ressaltar que os resultados apresentados na Tabela A.1 estão em consonância com os de Gomes (2004).

Apesar da evidência contrária à HRP apresentada até agora, nos exercícios seguintes são avaliados os efeitos de possíveis mudanças estruturais pelas quais a economia brasileira passou sobre a significância das estatísticas BDS. Particularmente, duas importantes quebras são consideradas: i) a queda na tendência secular de crescimento nos anos de 1980, período conhecido como a Década Perdida; e ii) a redução da instabilidade econômica ocorrida após o Plano Real.

A primeira quebra citada também é verificada para o consumo agregado anual das famílias no Brasil. Após realizar uma regressão do tipo $\Delta \log \left(C_{\mathrm{t}}\right)=c+\varepsilon_{\mathrm{t}}$ e implementar o teste de quebras múltiplas de Bai e Perron (1998) e a metodologia de Andrews (1993), a data de 1981 foi obtida como representativa da quebra na taxa de crescimento da variável a $1 \%$ de significância estatística. Dessa forma, o crescimento médio do consumo familiar entre 1947 e 1980 foi, em média, de 7\% ao ano. Ao passo que, entre 1981 e 2012, a taxa média é estimada em $2,5 \%$ ao ano.

Para levar em conta o resultado dos testes de quebras apresentados anteriormente, a Tabela 2 traz as estatísticas BDS para a série da primeira diferença do log-consumo trimestral, que varia entre 1991 e 2014. Como é dologias, consultar Nakamura e Nakamura (1981). 
possível verificar por esta tabela, o grande número de estatísticas BDS significativas a $1 \%$ conduz à rejeição da HRP para o caso da amostra completa de dados trimestrais. Nota-se, ainda, que a série trimestral não apresenta quebra estrutural na taxa de crescimento, segundo os testes de Bai e Perron (1998) e Andrews (1993).

Tabela 2 Teste de independência dos resíduos da primeira diferença da série do log-consumo, dados trimestrais, 1991 - 2014

\begin{tabular}{|c|c|c|c|c|c|c|c|c|c|c|c|c|}
\hline \multirow{2}{*}{$\boldsymbol{m}$} & \multicolumn{3}{|c|}{$\epsilon=0,5 \sigma_{c}$} & \multicolumn{3}{|c|}{$\epsilon=1 \sigma_{c}$} & \multicolumn{3}{|c|}{$\epsilon=1,5 \sigma_{c}$} & \multicolumn{3}{|c|}{$\epsilon=2 \sigma_{c}$} \\
\hline & $B D S$ & $P_{n}$ & $\boldsymbol{P}_{b}$ & $B D S$ & $P_{n}$ & $P_{b}$ & $B D S$ & $\boldsymbol{P}_{n}$ & $\boldsymbol{P}_{b}$ & $B D S$ & $P_{n}$ & $\boldsymbol{P}_{b}$ \\
\hline 2 & 3,7 & 0,00 & 0,00 & 4,3 & 0,00 & 0,00 & 4,8 & 0,00 & 0,00 & 4,6 & 0,00 & 0,00 \\
\hline 3 & 4,8 & 0,00 & 0,00 & 4,9 & 0,00 & 0,00 & 4,8 & 0,00 & 0,00 & 4,7 & 0,00 & 0,00 \\
\hline 4 & 5,0 & 0,00 & 0,01 & 5,2 & 0,00 & 0,00 & 5,1 & 0,00 & 0,00 & 5,1 & 0,00 & 0,00 \\
\hline 5 & 4,9 & 0,00 & 0,02 & 5,5 & 0,00 & 0,00 & 5,2 & 0,00 & 0,00 & 5,2 & 0,00 & 0,00 \\
\hline 6 & 4,8 & 0,00 & 0,03 & 5,6 & 0,00 & 0,00 & 5,3 & 0,00 & 0,00 & 5,1 & 0,00 & 0,00 \\
\hline
\end{tabular}

Fonte: Resultados da pesquisa.

Nota: 94 observações; $m$ representa a dimensão de imersão; BDS, a estatística de teste padronizada; $\mathrm{P}_{\mathrm{n}}$, o p-valor da distribuição normal; $\mathrm{P}_{\mathrm{b}}$, o -valor gerado por 10.000 simulações bootstrap; e $\sigma_{c}$ refere-se ao desvio padrão estimado do consumo.

Outra importante mudança recente foi a redução da volatilidade da atividade econômica ocorrida após a implantação do Plano Real. A Tabela 3 apresenta os testes BDS para a série das primeiras diferenças do log-consumo na amostra de 1996 a 2014 e de 1997 a 2014, datas que estão de acordo com as obtidas após aplicar os testes de Bai e Perron (1998) e Andrews (1993) à equação dos desvios quadrados do log-consumo contra uma constante (os testes indicaram quebra na volatilidade no segundo trimestre de 1997).

Pode-se perceber pelo painel $i$ da Tabela 3 que as estatísticas BDS continuam altamente significativas, tanto pela distribuição normal, como pelas simulações bootstrap. Ao considerar a quebra da volatilidade em 1997, no painel ii, contudo, grande parte da dependência temporal dos desvios da série do log-consumo desaparece. Existem evidências contrárias à hipótese IID nesta última amostra, principalmente quando $\epsilon=\sigma_{c}$, mas ela é menos contundente. Em consequência, o consumo dos agentes parece responder de maneira diversa conforme a volatilidade da economia se altera. 
Tabela 3 Teste de independência dos resíduos da primeira diferença da série do log-consumo, dados trimestrais

\begin{tabular}{|c|c|c|c|c|c|c|c|c|c|c|c|c|}
\hline \multicolumn{13}{|c|}{ Painel i - 1996 a 2014} \\
\hline \multirow{2}{*}{$M$} & \multicolumn{3}{|c|}{$\epsilon=0,5 \sigma_{c}$} & \multicolumn{3}{|c|}{$\epsilon=1 \sigma_{c}$} & \multicolumn{3}{|c|}{$\epsilon=1,5 \sigma_{c}$} & \multicolumn{3}{|c|}{$\epsilon=2 \sigma_{c}$} \\
\hline & BDS & $P_{n}$ & $P_{b}$ & $B D S$ & $\boldsymbol{P}_{n}$ & $P_{b}$ & BDS & $P_{n}$ & $P_{b}$ & $B D S$ & $P_{n}$ & $P_{b}$ \\
\hline 2 & 1,5 & 0,14 & 0,20 & 2,3 & 0,02 & 0,05 & 2,6 & 0,01 & 0,03 & 2,9 & 0,00 & 0,03 \\
\hline 3 & 1,7 & 0,09 & 0,18 & 3,0 & 0,00 & 0,02 & 2,9 & 0,00 & 0,02 & 2,6 & 0,01 & 0,04 \\
\hline 4 & 1,8 & 0,07 & 0,18 & 3,2 & 0,00 & 0,01 & 3,3 & 0,00 & 0,01 & 2,8 & 0,01 & 0,03 \\
\hline 5 & 2,7 & 0,01 & 0,12 & 3,7 & 0,00 & 0,01 & 3,6 & 0,00 & 0,00 & 3,1 & 0,00 & 0,02 \\
\hline 6 & 3,0 & 0,00 & 0,15 & 3,7 & 0,00 & 0,02 & 3,7 & 0,00 & 0,00 & 3,2 & 0,00 & 0,02 \\
\hline \multicolumn{13}{|c|}{ Painel ii - 1997 a 2014} \\
\hline \multirow{2}{*}{$M$} & \multicolumn{3}{|c|}{$\epsilon=0,5 \sigma_{c}$} & \multicolumn{3}{|c|}{$\epsilon=1 \sigma_{c}$} & \multicolumn{3}{|c|}{$\epsilon=1,5 \sigma_{c}$} & \multicolumn{3}{|c|}{$\epsilon=2 \sigma_{\mathrm{c}}$} \\
\hline & BDS & $P_{n}$ & $P_{b}$ & $B D S$ & $\boldsymbol{P}_{n}$ & $P_{b}$ & BDS & $\boldsymbol{P}_{n}$ & $P_{b}$ & $B D S$ & $P_{n}$ & $P_{b}$ \\
\hline 2 & 0,9 & 0,34 & 0,37 & 2,0 & 0,05 & 0,08 & 1,5 & 0,13 & 0,18 & 2,1 & 0,04 & 0,11 \\
\hline 3 & 1,3 & 0,20 & 0,28 & 2,2 & 0,03 & 0,06 & 1,6 & 0,10 & 0,14 & 1,8 & 0,07 & 0,14 \\
\hline 4 & 1,6 & 0,12 & 0,26 & 2,2 & 0,03 & 0,07 & 1,8 & 0,07 & 0,11 & 1,9 & 0,06 & 0,12 \\
\hline 5 & 2,8 & 0,01 & 0,14 & 2,7 & 0,01 & 0,04 & 2,1 & 0,03 & 0,07 & 2,0 & 0,05 & 0,10 \\
\hline 6 & 2,9 & 0,00 & 0,20 & 2,7 & 0,01 & 0,05 & 2,2 & 0,03 & 0,06 & 1,8 & 0,07 & 0,12 \\
\hline
\end{tabular}

Fonte: Resultados da pesquisa.

Nota: 94 observações; $m$ representa a dimensão de imersão; BDS, a estatística de teste padronizada; $\mathrm{P}_{n}$, o $p$-valor da distribuição normal; $\mathrm{P}_{\mathrm{b}}$, o $p$-valor gerado por 10.000 simulações bootstrap; e $\sigma_{c}$ refere-se ao desvio padrão estimado do consumo.

A Tabela 4, a seguir, traz os resultados das estimações do modelo com restrições ao crédito, descrito na equação (7). A lista de instrumentos está descrita nas notas explicativas da tabela, seguindo o proposto por Ludvigson (1999). Percebe-se que o parâmetro $\lambda$ é sempre significativo e elevado. A média de suas estimativas está próxima de 0,75 , inferior ao valor obtido anteriormente, de 0,90 , quando a restrição ao crédito não foi controlada (ver Tabela A.1). O coeficiente associado às variações percentuais do volume de crédito, quando significativo, é estimado em 0,10 , aproximadamente, valor muito próximo ao encontrado por Ludvigson (1999). Portanto, um aumento totalmente antecipado de $10 \%$ na quantidade de crédito concedido ao setor privado gera uma elevação de $1 \%$ na taxa de crescimento trimestral do consumo agregado brasileiro. Além disso, nota-se que o valor do coeficiente estimado para a taxa de juros real foi pequeno e não significativo, em concordância com a litera- 
tura prévia nacional e internacional. Como explicado por Gomes (2010), a não rejeição da hipótese nula $\theta=0$ fornece evidências contra a HRP em formulações teóricas mais gerais do que aquela desenvolvida na segunda seção desta pesquisa. ${ }^{11}$

Tabela 4 Hipótese de restrição à liquidez de Ludvigson (1999)

Série trimestral, $1991-2012$

\begin{tabular}{|c|c|c|c|c|}
\hline Instrumentos & Estimativa de $\lambda$ & Estimativa de $\theta$ & Estimativa de $\alpha$ & Estatística F \\
\hline Conjunto 1 & $* * 0,74$ & - & - & $* * 4,61$ \\
\hline Conjunto 2 & - & - & $* 0,10$ & $* * 6,56$ \\
\hline Conjunto 3 & ${ }^{*} 0,91$ & $0,0002^{N S}$ & - & $* * 4,75$ \\
\hline Conjunto 4 & ${ }^{*} 0,88$ & $0,0006^{N S}$ & $0,02^{N S}$ & $* 4,59$ \\
\hline Conjunto 5 & $* * * 0,67$ & $-0,0001^{N S}$ & $0,04^{N S}$ & $* * 4.03$ \\
\hline Conjunto 6 & ${ }^{* *} 0,72$ & $-0,0001^{N S}$ & $* * * 0,12$ & $* 5,87$ \\
\hline Conjunto 7 & ${ }^{*} 0,70$ & $0,0006^{N S}$ & $0,05^{N S}$ & $* 6,70$ \\
\hline Conjunto 8 & ${ }^{* *} 0,65$ & $-0.0004^{N S}$ & $* * * 0,089$ & *7,25 \\
\hline Conjunto 9 & *0,77 & $-0,0001^{N S}$ & $* * * 0,10$ & *6,66 \\
\hline Média & 0,76 & 0,0001 & 0,07 & - \\
\hline
\end{tabular}

Notas: Todas estatísticas DWH não significativas a 10\%. ${ }^{*},{ }^{* *} e^{* * *}$ representam significância estatística aos niveis de 1, 5 e 10\%, respectivamente. NS denota não significativo. Conj. 1: defasagens 2 a 4 do crescimento do consumo e da renda e defasagem 2 do crescimento do juros; Conj. 2: defasagens 2 a 4 do crescimento da renda e da taxa de juros real em nível e defasagem dois do crescimento do juros; Conj. 3 : igual ao 1 mais defasagens 2 a 4 da taxa de juros real em nível; Conj. 4: defasagens 2 a 4 do crescimento do consumo, do crédito e da taxa de juros e defasagens 2 a 4 da taxa de juros real em nível; Conj. 5: defasagens 2 a 4 do crescimento do consumo e da renda e defasagens 2 a 4 do nível e do crescimento da taxa de juros real; Conj. 6: defasagens 2 a 4 do crescimento da renda, do crédito, do crescimento e do nível da taxa de juros real; Conj. 7: defasagens 2 a 5 do crescimento do consumo, da renda e do crédito, do nível e do crescimento da taxa de juros real; Conj. 8: defasagens 2 a 5 do crescimento da renda e do crédito, do nível e do crescimento da taxa de juros real; Conj. 9: igual ao 6 mais defasagens 2 a 4 do termo de correção (diferença do logaritmo do consumo e da renda).

É interessante destacar que, mesmo $\lambda$ tendo sido reduzido após a introdução da taxa de crescimento do crédito no modelo econométrico, seu valor

11 Uma ressalva importante deve ser feita a respeito dos resultados apresentados na Tabela 4: Gomes e Paz (2013; 2015) discutem os efeitos da correta instrumentalização em equações como (7), particularmente no caso da variável taxa de juros, que deve representar adequadamente a taxa de retorno do portfólio do agente representativo e está relacionada com a elasticidade de substituição intertemporal do consumo. Gomes e Paz (2015), por exemplo, encontram evidências de que os instrumentos propostos por Mulligan (2002) não apresentam o problema de instrumentos fracos e proporcionam estimativas significativas e maiores que a unidade para a variável associada à elasticidade de substituição. 
médio em torno de 0,70 ou 0,75 , estatisticamente diferente de zero, indica que existem explicações adicionais para a alta sensibilidade do consumo às variações previstas da renda corrente. Adicionalmente, esse resultado mostra que as políticas que facilitam o acesso ao crédito podem melhorar o bem-estar de fração considerável da população brasileira.

\subsection{Teste da função consumo com excesso de sensibilidade à ren- da corrente}

Verifica-se neste momento a adequabilidade da função consumo apresentada nas equações (8) e (9) aos dados brasileiros. Para tanto, são estimados modelos de correção do erro, utilizando as séries anuais do consumo familiar e da renda, descritas anteriormente e ilustradas na Figura 1, partes $a$ e $b$. Regressões do tipo MCE são aplicáveis quando os dados exibem uma mesma ordem de integração. Se as séries estudadas são integradas de ordem $d$, isto é, $I(d)$, e têm uma tendência comum de longo prazo, sendo cointegradas, então Engle e Granger (1987) mostram que uma combinação linear destas séries é integrada de ordem zero, $I(0)$, ou seja, estacionária. Caso contrário, a regressão entre o consumo e a renda em nível apresentaria problemas de regressão espúria.

Dessa forma, é preciso testar se o consumo e a renda têm uma raiz unitária, sendo, na terminologia de Engle e Granger (1987), caracterizados como processos I(1). As saídas dos testes ADF de Dickey e Fuller (1979) e de Zivot e Andrews (1992), que têm maior poder na possibilidade de quebras estruturais, são apresentadas na Tabela A.2 do anexo. Como é possível verificar, existem fortes indícios de que as séries do consumo e da renda em nível no Brasil apresentam uma raiz unitária, mesmo após considerar a possibilidade de quebras nos parâmetros. Resultados similares foram encontrados por Nelson e Plosser (1982), para o caso dos EUA, e por Issler e Rocha (2000) e Gomes (2004), para o caso do Brasil.

Prosseguindo com o teste de cointegração, a relação de longo prazo foi estimada pelo método de mínimos quadrados. Em seguida, foram obtidos os resíduos da regressão, sobre os quais foi aplicado o teste de raiz unitária sem termos exógenos na equação de teste. Os valores críticos são os de Engle e Granger (1987). Os resultados desse procedimento estão resumidos na Tabela A.3. Verifica-se que a estatística de teste permite rejeitar a 
hipótese nula de raiz unitária a 5\% de significância, o que possibilita a estimação do modelo de correção do erro. ${ }^{12}$

O modelo de correção do erro sem quebras estruturais é exibido na Tabela 5. De acordo com as estimativas obtidas, a propensão marginal do consumo em longo prazo, $b$, foi de 0,9 , valor significativo ao nível de $1 \%$, bastante similar ao obtido para a elasticidade de curto prazo, $\beta_{1}$. 0 coeficiente que aparece na variável de correção do erro, $\gamma$, é negativo e significativo a $1 \%$, indicando presença de ajustamentos defasados nos gastos com consumo. Por fim, os demais coeficientes não foram estatisticamente diferentes de zero ao nível de 10\%, inclusive o relacionado com a hipótese de formação de hábitos.

Tabela 5 Resultados do modelo de correção do erro, sem quebras estruturais

\begin{tabular}{|c|c|c|c|c|}
\hline \multicolumn{5}{|c|}{ Equação de longo prazo: $C_{t}=\alpha+b Y_{t}+e_{t}$} \\
\hline Parâmetro & Estimativa & Erro padrão & Estatística $t$ & $p$-valor \\
\hline$A$ & $-0,318$ & 0,008 & $-39,1$ & 0,000 \\
\hline$B$ & 0,934 & 0,005 & 160,2 & 0,000 \\
\hline$R^{2}$ & 0,998 & & & \\
\hline$R^{2}$ ajustado & 0,997 & & & \\
\hline$F$ & 25661 & & & 0,000 \\
\hline Durbin-Watson & 0,487 & & & \\
\hline \multicolumn{5}{|c|}{ Equação de curto prazo: $\Delta C_{t}=\beta_{0}+\beta_{1} \Delta Y_{t}+\beta_{2} \Delta Y_{t-1}+\beta_{3} \Delta C_{t-1}+\gamma e_{t-1}+u_{t}$} \\
\hline Parâmetro & Estimativa & Erro padrão & Estatística $t$ & $p$-valor \\
\hline$\beta_{0}$ & -0.001 & 0.007 & -0.187 & 0.852 \\
\hline$\beta_{1}$ & 0.932 & 0.111 & 8.375 & 0.000 \\
\hline$\beta_{2}$ & -0.071 & 0.165 & -0.431 & 0.668 \\
\hline$\beta_{3}$ & 0.109 & 0.130 & 0.839 & 0.405 \\
\hline$\Gamma$ & -0.271 & 0.090 & -2.996 & 0.004 \\
\hline$R^{2}$ & 0,631 & & & \\
\hline$R^{2}$ ajustado & 0,606 & & & \\
\hline$F$ & 25,216 & & & 0,000 \\
\hline Durbin-Watson & 1,984 & & & \\
\hline
\end{tabular}

Fonte: Resultados da pesquisa. As variáveis consumo e renda estão em logaritmos.

12 Resultados similares são obtidos ao aplicar os testes de traço e autovalor de Johansen (1995) aos dados, que indicam a existência de uma relação de cointegração quando a relação de longo prazo assume o formato da função consumo exibida na equação (8). 
Na Tabela 6, a hipótese IID para os resíduos do mecanismo de correção do erro é avaliada. Como é possível verificar, há um elevado número de estatísticas BDS significativas, seja sob a suposição de normalidade, seja adotando a distribuição simulada. Assim, pode-se rejeitar a hipótese de que o modelo representado na Tabela 5 seja representativo dos dados brasileiros. Inclusive, esta regressão pode estar mal especificada, haja vista que as propensões a consumir em longo e curto prazo foram praticamente iguais, quando, na verdade, se espera um valor menor para a segunda (veja-se os valores de $b$ e $\beta_{1}$ ). Dessa forma, prossegue-se com a verificação da adequabilidade das funções consumo ao permitir quebras estruturais nos parâmetros do modelo representado pelas equações (8) e (9).

Tabela 6 Teste de independência dos resíduos do modelo de correção do erro, dados anuais, $1947-2012$

\begin{tabular}{|c|c|c|c|c|c|c|c|c|c|c|c|c|}
\hline \multirow{2}{*}{$m$} & \multicolumn{3}{|c|}{$\epsilon=0,5 \sigma_{c}$} & \multicolumn{3}{|c|}{$\epsilon=1 \sigma_{c}$} & \multicolumn{3}{|c|}{$\epsilon=1,5 \sigma_{c}$} & \multicolumn{3}{|c|}{$\epsilon=2 \sigma_{c}$} \\
\hline & $B D S$ & $\boldsymbol{P}_{n}$ & $P_{b}$ & $B D S$ & $\boldsymbol{P}_{n}$ & $\boldsymbol{P}_{b}$ & $B D S$ & $P_{n}$ & $\boldsymbol{P}_{b}$ & BDS & $P_{n}$ & $P_{b}$ \\
\hline 2 & 4,0 & 0,00 & 0,05 & 2,5 & 0,01 & 0,05 & 1,9 & 0,05 & 0,10 & 3,8 & 0,00 & 0,01 \\
\hline 3 & 3,5 & 0,00 & 0,10 & 2,7 & 0,01 & 0,05 & 2,0 & 0,04 & 0,09 & 4,0 & 0,00 & 0,01 \\
\hline 4 & 4,0 & 0,00 & 0,11 & 1,8 & 0,06 & 0,14 & 1,2 & 0,22 & 0,23 & 3,4 & 0,00 & 0,02 \\
\hline 5 & 0,9 & 0,37 & 0,61 & 1,6 & 0,11 & 0,19 & 0,9 & 0,38 & 0,34 & 3,1 & 0,00 & 0,03 \\
\hline 6 & 3,3 & 0,00 & 0,33 & 1,8 & 0,08 & 0,17 & 0,6 & 0,55 & 0,45 & 2,7 & 0,01 & 0,05 \\
\hline
\end{tabular}

Fonte: Resultados da pesquisa.

Nota: 66 observações; $m$ representa a dimensão de imersão; BDS, a estatística de teste padronizada; $\mathrm{P}_{\mathrm{n}}$, o $\mathrm{p}$-valor da distribuição normal; $\mathrm{P}_{\mathrm{b}}$, o $p$-valor gerado por 10.000 simulações bootstrap; e $\sigma_{\mathrm{c}}$ refere-se ao desvio padrão estimado do consumo.

A próxima etapa metodológica é reavaliar o MCE considerando a possiblidade de quebras estruturais para melhorar a especificação das regressões estimadas. Com esse intuito, utiliza-se o teste de Gregory e Hansen (1996), que permite verificar se as séries da renda e do consumo são não cointegradas, contra a alternativa de cointegração com uma possível mudança (quebra) no intercepto e na inclinação da relação de longo prazo. A data da quebra é desconhecida a priori, sendo definida no ponto do tempo em que a estatística de teste é maximizada, de forma a aumentar as chances de rejeição da hipótese nula. Valores críticos foram simulados pelos autores e, como é comum nas aplicações da área, adota-se o valor de $15 \%$ para o 
parâmetro de trimming. A Tabela A.4 resume os resultados da aplicação do teste de cointegração com quebras.

Veja-se que mesmo após considerar a existência de uma quebra estrutural na relação entre as variáveis, estimada em 1985, é possível rejeitar a hipótese nula de não cointegração a 10\% de significância. Assim, pode-se afirmar com certa segurança que a renda corrente e o consumo mantêm uma relação de equilíbrio de longo prazo, o que, por si só, é uma evidência contrária à hipótese da renda permanente de Hall (1978).

Em razão da mudança averiguada nos parâmetros da relação de cointegração, estima-se o MCE permitindo quebras estruturais nas equações de longo e de curto prazo. Utiliza-se, para tanto, o método sequencial de Bai e Perron (1998), em que, após escolher um nível de significância estatística, por exemplo, de $5 \%$, testa-se a hipótese de estabilidade nos parâmetros contra a alternativa de uma quebra. Em seguida, existindo uma quebra estrutural, outras são procuradas dentro das subamostras geradas. $\mathrm{O}$ algoritmo termina quando se alcança o número máximo de quebras, definido previamente, ou quando as datas estimadas são não significativas. A Tabela 7 mostra os resultados do método. ${ }^{13}$

Nota-se que o método de Bai e Perron (1998) estimou a quebra significativa a 5\% na relação de cointegração em 1987, valor muito próximo daquele exibido na Tabela A.4. Nas duas subamostras geradas, o valor da elasticidade de longo prazo do consumo à renda é alto. Contudo, os resultados obtidos para a segunda amostra são bastante interessantes, visto que, nesse período, consumo e renda corrente são praticamente proporcionais, indicando que o consumo no Brasil responde fortemente às variações nesta variável.

Com respeito à equação de curto prazo, o método detectou apenas uma quebra estrutural, ocorrida em 1988, ou seja, quase na mesma data obtida para a mudança na relação de cointegração. Ao comparar as duas amos-

13 Para manter a conformidade entre o teste de cointegração com quebras estruturais e os resultados apresentados na Tabela 7, na relação de cointegração entre renda e consumo foi definido o número máximo de uma quebra, enquanto na equação de curto prazo foi permitida a ocorrência de até cinco mudanças estruturais. A estimação do MCE com quebras segue um processo sequencial. Primeiro, o algoritmo verifica a existência de quebras na relação de longo prazo e, uma vez encontrada, utilizam-se todos os dados para a estimação dos parâmetros em cada período. Em seguida, os resíduos da primeira etapa são obtidos e utilizados como uma variável adicional na relação de curto prazo. Por fim, o método de Bai e Perron (1998) procura novas datas de quebras na regressão de curto prazo, que tem os parâmetros referentes a cada amostra estimados conjuntamente, utilizando as séries de tempo por completo. 
tras, percebe-se uma expressiva redução na propensão a consumir de curto prazo, que passou de 1,00 entre 1947 e 1987, para 0,37 entre 1988 e 2012. O último valor está próximo aos encontrados por Mehra (2001).

Tabela 7 Saídas do modelo de correção do erro, com quebras estruturais

\begin{tabular}{|c|c|c|c|c|}
\hline \multicolumn{5}{|c|}{ Equação de longo prazo: $C_{t}=\alpha+b Y_{t}+e_{t}$} \\
\hline Parâmetro & Estimativa & Erro padrão & Estatística $t$ & $p$-valor \\
\hline \multicolumn{5}{|l|}{1947 - 1986} \\
\hline$A$ & $-0,318$ & 0,006 & $-53,65$ & 0,000 \\
\hline$B$ & 0,97 & 0,007 & 146,62 & 0,000 \\
\hline \multicolumn{5}{|l|}{$1987-2012$} \\
\hline$A$ & $-0,47$ & 0,058 & $-8,11$ & 0,000 \\
\hline$B$ & 1,00 & 0,007 & 33,11 & 0,000 \\
\hline$R^{2}$ & 0,999 & & & \\
\hline$R^{2}$ ajustado & 0,999 & & & \\
\hline$F$ & 16237 & & & 0,000 \\
\hline Durbin-Watson & 0,835 & & & \\
\hline \multicolumn{5}{|c|}{ Equação de curto prazo: $\Delta C_{t}=\beta_{0}+\beta_{1} \Delta Y_{t}+\beta_{2} \Delta Y_{t-1}+\beta_{3} \Delta C_{t-1}+\gamma e_{t-1}+u_{t}$} \\
\hline Parâmetro & Estimativa & Erro padrão & Estatística $t$ & p-valor \\
\hline \multicolumn{5}{|l|}{$1947-1987$} \\
\hline$\beta_{0}$ & 0.003 & 0.009 & 0.357 & 0.723 \\
\hline$\beta_{1}$ & 1.003 & 0.125 & 8.036 & 0.000 \\
\hline$\beta_{2}$ & -0.152 & 0.247 & -0.617 & 0.540 \\
\hline$\beta_{3}$ & 0.014 & 0.186 & 0.074 & 0.942 \\
\hline$\gamma$ & -0.669 & 0.213 & -3.138 & 0.003 \\
\hline \multicolumn{5}{|l|}{$1988-2012$} \\
\hline$\beta_{0}$ & 0.007 & 0.009 & 0.803 & 0.425 \\
\hline$\beta_{1}$ & 0.373 & 0.195 & 1.910 & 0.061 \\
\hline$\beta_{2}$ & -0.165 & 0.213 & -0.775 & 0.442 \\
\hline$\beta_{3}$ & 0.603 & 0.161 & 3.741 & 0.000 \\
\hline$\gamma$ & -0.374 & 0.133 & -2.803 & 0.007 \\
\hline$R^{2}$ & 0,764 & & & \\
\hline$R^{2}$ ajustado & 0,725 & & & \\
\hline$F$ & 19,45 & & & 0,000 \\
\hline Durbin-Watson & 1,802 & & & \\
\hline
\end{tabular}

Fonte: Resultados da pesquisa. As variáveis consumo e renda estão em logaritmos. 
A análise com quebras estruturais fornece outro resultado importante. Nota-se que na segunda amostra o coeficiente do consumo defasado se torna estatisticamente significativo, mostrando que a hipótese de formação de hábito é adequada ao período. A formação de hábitos pode ser explicada pelas diversas mudanças passadas pela economia brasileira no período pós 1988. Entre esse ano e 1994, por exemplo, tal comportamento pode estar associado com a prática de consumir rapidamente a renda corrente para evitar as perdas advindas da hiperinflação. Por outro lado, desde 1995 a maior estabilidade da economia pode ser um fator explicativo da formação de hábitos. Políticas como a valorização do câmbio nos anos iniciais do Plano Real e os recentes estímulos ao consumo via reduções de impostos também podem ter contribuído para a significância do consumo defasado.

Ressalta-se que no segundo período a velocidade do ajuste dos desvios do consumo em torno da relação de longo prazo, $\gamma$, se reduziu consideravelmente, passando de 0,669 para 0,374 em valores absolutos. Novamente, a maior estabilidade da economia pode explicar esses números, pois erros de planejamento em situações normais da economia tendem a ser menos custosos.

Em suma, os resultados obtidos pelo MCE com quebras estruturais indicam que a dinâmica do consumo agregado brasileiro foi alterada significativamente nos anos de 1987 e 1988. Assim, na Tabela 8 a seguir, examina-se a adequabilidade deste modelo aos dados brasileiros.

Pela análise da Tabela 8, é possível verificar que todas as estatísticas BDS são não significativas a $10 \%$, o que leva à conclusão de que o modelo MCE com quebras estruturais representa satisfatoriamente o consumo agregado no Brasil, constatação que gera implicações teóricas e práticas para a literatura econômica no país. Por exemplo, dado que a proporção dos agentes que respondem aos movimentos da renda corrente é muito alta, é preciso aprofundar o entendimento a respeito do peso relativo de cada tipo de restrição, ao crédito ou voluntárias, sobre esse comportamento. A solução de problemas dessa natureza é essencial, por exemplo, para estimular apropriadamente a poupança. Ademais, a sensibilidade do consumo à renda corrente abre espaço para políticas de estabilização das flutuações econômicas. Cunha (2004) estimou elevadas perdas de bem-estar nas recessões no Brasil, o que torna as políticas anticíclicas desejáveis e, conforme indicam as estimativas obtidas pelo presente estudo, potencialmente efetivas. 
Tabela 8 Teste de independência dos resíduos do modelo de correção do erro com quebras estruturais, dados anuais, 1947 - 2012

\begin{tabular}{|c|c|c|c|c|c|c|c|c|c|c|c|c|}
\hline \multirow{2}{*}{$m$} & \multicolumn{3}{|c|}{$\epsilon=0,5 \sigma_{c}$} & \multicolumn{3}{|c|}{$\epsilon=1 \sigma_{c}$} & \multicolumn{3}{|c|}{$\epsilon=1,5 \sigma_{c}$} & \multicolumn{3}{|c|}{$\epsilon=2 \sigma_{c}$} \\
\hline & BDS & $\boldsymbol{P}_{n}$ & $P_{b}$ & $B D S$ & $\boldsymbol{P}_{n}$ & $P_{b}$ & $B D S$ & $P_{n}$ & $\boldsymbol{P}_{b}$ & BDS & $\boldsymbol{P}_{n}$ & $P_{b}$ \\
\hline 2 & $-0,2$ & 0,83 & 1,00 & 0,0 & 0,98 & 0,83 & 0,7 & 0,50 & 0,51 & 0,4 & 0,68 & 0,62 \\
\hline 3 & $-0,4$ & 0,69 & 0,92 & 0,5 & 0,63 & 0,53 & 0,2 & 0,81 & 0,70 & 0,7 & 0,49 & 0,48 \\
\hline 4 & $-1,0$ & 0,33 & 0,63 & 0,3 & 0,78 & 0,62 & $-0,3$ & 0,74 & 0,98 & 0,5 & 0,63 & 0,57 \\
\hline 5 & $-0,3$ & 0,75 & 0,93 & 0,2 & 0,82 & 0,63 & 0,0 & 0,98 & 0,82 & 0,5 & 0,64 & 0,56 \\
\hline 6 & 1,1 & 0,25 & 0,42 & 0,4 & 0,70 & 0,53 & 0,1 & 0,95 & 0,74 & 0,3 & 0,74 & 0,63 \\
\hline
\end{tabular}

Fonte: Resultados da pesquisa.

Nota: 66 observações; $m$ representa a dimensão de imersão; BDS, a estatística de teste padronizada; $\mathrm{P}_{\mathrm{n}}$, o $\mathrm{p}$-valor da distribuição normal; $\mathrm{P}_{\mathrm{b}}$, o $p$-valor gerado por 10.000 simulações bootstrap; e $\sigma_{\mathrm{c}}$ refere-se ao desvio padrão estimado do consumo.

\section{Conclusão}

O presente artigo objetivou avaliar a adequação de teorias para o consumo aos dados brasileiros. Especificamente, a hipótese da renda permanente (HRP) e a função consumo com alta sensibilidade à renda corrente foram analisadas com base no teste BDS, de Brock et al. (1996). Conforme os resultados obtidos mostraram, a HRP foi rejeitada, isto é, os resíduos da primeira diferença das séries do consumo não se comportaram como um passeio aleatório, mesmo quando foram consideradas as mudanças estruturais ocorridas em 1980, como a quebra na tendência de crescimento de longo prazo da economia, e após o Plano Real, quando a volatilidade se reduziu.

De fato, estimativas inspiradas pelo método desenvolvido por Campbell e Mankiw (1990) indicaram que os consumidores que detêm 90\% da renda nacional não se comportam segundo as previsões da HRP. Com a introdução do volume de crédito nas estimações, o percentual da renda que desvia desta hipótese se reduz para 75 ou $70 \%$, em média, indicando que a restrição à liquidez, apesar de ser um importante determinante da alta correlação entre o consumo e a renda corrente no Brasil, não é o único. Portanto, o consumo de uma fração significativa da população brasileira responde às variações previstas da renda.

Dessa forma, procurou-se representar a relação entre consumo e renda corrente por meio do mecanismo de correção do erro (MCE) de Engle e 
Granger (1987), utilizando dados anuais entre 1947 e 2012. Não foi possível rejeitar a hipótese de cointegração entre consumo e renda corrente no país, mesmo na presença de quebras estruturais. Ademais, o MCE com quebras obteve resíduos do tipo ruído branco, segundo o teste BDS, validando a adequabilidade do modelo com sensibilidade à renda corrente aos dados.

Além disso, os resultados mostraram que após o ano de 1988 se detecta uma tendência à formação de hábitos de consumo no país. Ao final da década de 1980, estimou-se um coeficiente autorregressivo da ordem de 0,6 para as variações defasadas do consumo agregado, o qual pode estar associado ao alto consumo do período hiperinflacionário e à estabilidade alcançada pela economia nos anos mais recentes da amostra.

Nesse sentido, a principal conclusão obtida é que o consumo agregado no Brasil é bem caracterizado como sendo dependente das variações previstas da renda. Especificamente, não se rejeita a hipótese de que um modelo simples de correção do erro com quebras estruturais seja capaz de representar toda a dependência temporal dos dados. Consequentemente, as políticas fiscais e monetárias podem ser instrumentos eficazes de suavização dos ciclos econômicos. Como existe uma alta sensibilidade do consumo à renda corrente, abre-se espaço para as políticas governamentais que visem a amenizar as flutuações ocorridas na atividade econômica.

Como indicações de pesquisas futuras, sugere-se investigar a seguinte questão: por que a hipótese de sensibilidade do consumo à renda corrente é a adequada? Ou, dito de outra forma, por que os consumidores são sensíveis às variações da renda corrente no Brasil? O mecanismo de correção do erro, como uma função em forma reduzida, pode ser derivado de diferentes especificações, por exemplo, assumindo-se restrições à liquidez (como em Ludvigson, 1999; ou Deaton, 1989), restrições comportamentais (como em Thaler, 1990) ou a própria regra psicológica de Keynes (1936). Acredita-se que a discriminação da importância relativa de cada um desses fatores, entre outros possíveis, sobre o comportamento do consumidor, é uma linha de estudo de relevância. Assim, o direcionamento do foco das análises para o nível microeconômico poderá enriquecer o entendimento atual a respeito da dinâmica do consumo agregado. 


\section{Referências}

AKAIKE, H. Factor Analysis and AIC. Psychometrika, v. 52, n. 3, p. 317-332, 1987.

ANDREWS, D. Tests for Parameter Instability and Structural Change With Unknown Change Point. Econometrica, v. 61, n. 4, p. 821-856, 1993.

ATTANASIO, O. P.; WEBER, G. Consumption and saving: models of intertemporal allocation and their implications for public Policy. Journal of Economic Literature, v. 48, n. 3, p. 693-751, 2010.

BAI, L.; PERRON, P. Estimating and testing linear models with multiple structural changes. Econometrica, v. 66, n. 1, p. 47-78, 1998.

BARNETT, W.; SERLETIS, A. Martingales, nonlinearity, and chaos. Journal of Economic Dynamics and Control, v. 24, n. 5, p. 703-724, 2000.

BONELLI, R.; RODRIGUES, C. PIB trimestral: proposta metodológica e resultados para o período 1947-79. Texto para discussão n. 3. Rio de Janeiro: FGV, 2012.

BROCK, W.; SCHEINKMAN, J.; DECHERT, W.; LEBARON, B. A test for independence based on the correlation dimension. Econometric reviews, v. 15, n. 3, p. 197-235, 1996.

BROWNING, M.; CROSSLEY, T. F. The life-cycle model of consumption and saving. Journal of Economic Perspectives, v. 15, n. 3, p.3-22, 2001.

CAMPBELL, J.; MANKIW, N. Permanent income, current income, and consumption. Journal of Business \& Economic Statistics, v. 8, n. 3, p. 265-279, 1990.

CHEN, S.; YEH, C. On the emergent properties of artificial stock markets: The efficient market hypothesis and the rational expectations hypothesis. Journal of Economic Behavior \& Organization, v. 49, n. 2, p.217-239, 2002.

CUNHA, B.; FERREIRA, P. Custo de Ciclo Econômico no Brasil em um Modelo com Restrição a Crédito. Estudos Econômicos, v. 34, n. 2, p. 243-268, 2004.

DAVIDSON, J.; HENDRY, D.; SRBA, F.; YEO, S. Econometric modelling of the aggregate time-series relationship between consumers' expenditure and income in the United Kingdom. Economic Journal, v. 88, n. 352, p. 661-692, 1978.

DEATON, A. Saving in developing contries: Theory and review. Working paper 144, Departament of Economics, Princeton University, 1989.

DICKEY, D.; FULLER, W. Distribution of the estimators for autoregressive time series with a unit root. Journal of the American Statistical Association, v. 74, n. 366a, p. 427-431, 1979.

ENGLE, R.; GRANGER, C. Co-integration and error correction: Representation, estimation, and testing. Econometrica, v. 55, n. 2, p. 251-276, 1987.

FAMA, E. Efficient capital markets: Review of theory and empirical work. Journal of Finance, v. 25 , n. 2 , p. $383-417,1970$.

FLAVIN, M. The adjustment of consumption to changing expectations about future income. Journal of Political Economy, v. 89, n. 5, p. 974-1009, 1981.

FRIEDMAN, M. A Theory of the Consumption Function. Princeton, NJ: Princeton University Press, 1957. 
FUHRER, J. C. Habit formation in consumption and its implications for monetary-policy models. American Economic Review, v. 90, n. 3, p. 367-390, 2000.

GOMES, F. A. R. Consumo no Brasil: Teoria da renda permanente, formação de hábito e restrição à liquidez. Revista Brasileira de Economia, v. 58, n. 3, p. 381-402, 2004.

GOMES, F. A. R. Consumo no Brasil: Comportamento otimizador, restrição de crédito ou miopia? Revista Brasileira de Economia, v. 64, n. 3, p. 261-275, 2010.

GOMES, F. A. R.; PAZ, L. S. Especificações para a função consumo: Testes para países da América do Sul. Pesquisa e Planejamento Econômico, v. 34, n. 1, p. 39-55, 2004.

GOMES, F. A. R.; PAZ, L. S. Consumption in South America: myopia or liquidity constraints? Economia Aplicada, v. 14, n. 2, p. 129-145, 2010.

GREGORY, A.; HANSEN, B. Practitioners corner: Tests for cointegration in models with regime and trend shifts. Oxford bulletin of Economics and Statistics, v. 58, n. 3, p. 555-560, 1996.

HALL, R. Stochastic implications of the life-cycle Permanent Income Hypothesis: Theory and evidence. Journal of Political Economy, v. 86, n. 6, p. 971-987, 1978.

HALL, R.; MISHKIN, F. The sensitivity of consumption to transitory income: Estimates from panel data on households. Econometrica, v. 50, n. 2, p. 461-481, 1982.

HAYASHI, F. The Permanent Income Hypothesis: Estimation and testing by instrumental variables. Journal of Political Economy, v. 90, n. 5, p. 895-916, 1982.

HSIEH, D. A. Testing for nonlinear dependence in daily foreign exchange rates. Journal of Business, v. 62, n. 3, p. 339-68, 1989.

IBGE - Instituto Brasileiro de Geografia e Estatística. Sistema de Contas Nacionais - Brasil - referência 2000. Rio de Janeiro: IBGE, 2006. Disponível em: <http://www.ipeadata.gov.br/ Default.aspx>. Acesso em: 10 de julho de 2016.

ISSLER, J. V.; ROCHA, F. Consumo, restrição a liquidez e bem-estar no Brasil. Economia Aplicada, v. 4, n. 4, p. 637-665, 2000.

JOHANSEN, S. Likelihood-based inference in cointegrated vector autoregressive models. Oxford: Oxford University Press, 1995.

KEYNES, J. M. The general theory of employment, interest and money. Londres: MacMillan Cambridge University Press, 1936.

LUDVIGSON, S. Consumption and credit: A model of time-varying liquidity constraints. Review of Economics and Statistics, v. 81, n. 3, p. 434-447, 1999.

MACKINNON, J. Numerical distribution functions for unit root and cointegration tests. Journal of Applied Econometrics, v. 11, n. 6, p. 601-618, 1996.

MANKIW, N. G. Hall's consumption hypothesis and durable goods. Journal of Monetary Economics, v. 10, n. 3, p. 417-425, 1982.

MEHRA, Y. The wealth effect in empirical life-cycle aggregate consumption equations. Economic Quarterly-Federal Reserve Bank of Richmond, v. 87, n. 2, p.45-68, 2001.

MULLIGAN, C. Capital, interest, and aggregate intertemporal substitution. NBER Working Paper $n .9373,2002$.

NAGATANI, K. Life cycle saving: Theory and fact. American Economic Review, v. 62, n. 3, p. 
344-353, 1972.

NAKAMURA, A.; NAKAMURA, M. On the relationships among several specification error tests presented by Durbin, Wu, and Hausman. Econometrica, v. 49, n. 6, p.1583-1588, 1981.

NELSON, C.; PLOSSER, C. Trends and random walks in macroeconomic time series: Some evidence and implications. Journal of Monetary Economics, v. 10, n. 2, p. 139-162, 1982.

RAHMAN, A.; SAADI, S. Random walk and breaking trend in financial series: An econometric critique of unit root tests. Review of Financial Economics, v. 17, n. 3, p. 204-212, 2008.

REIS, E.; ISSLER, J. V.; BLANCO, F.; CARVALHO, L. Renda permanente e poupança precaucional: Evidências empíricas para o Brasil no passado recente. Pesquisa e Planejamento Econômico, v. 28, n. 2, p. 233-272, 1998.

ROMER, D. Advanced Macroeconomics (2 ed.). New York: McGraw-Hill, 2001.

SHEA, J. Myopia, liquidity constraints, and aggregate consumption: A simple test. Journal of Money, Credit and Banking, v. 27, n. 3, p. 798-805, 1995.

SHEFRIN, H.; THALER, R. The behavioral life-cycle hypothesis. Economic Inquiry, v. 26, n. 4, p. 609-643, 1988.

THALER, R. Anomalies: Saving, fungibility, and mental accounts. Journal of Economic Perspectives, v. 4, n. 1, p. 193-205, 1990.

THOMAS, J. The early econometric history of the consumption function. Oxford Economic Papers, v. 41, n. 1, p. 131-149, 1989.

WEBER, C. E. Intertemporal non-separability and "rule of thumb" consumption. Journal of Monetary Economics, v. 49, n. 2, p. 293-308, 2002.

ZIVOT, E.; ANDREWS, D. Further Evidence on the Great Crash, the Oil-Price Shock, and the Unit-Root Hypothesis. Journal of Business \& Economic Statistics, v. 10, n. 3, p. 251-270, 1992.

\section{Sobre o autor}

Luckas SabioniLopes-luckas.lopes@uff.edu.br

Universidade Federal de Juiz de Fora, campus de Governador Valadares, Minas Gerais.

\section{Sobre 0 artigo}

Recebido em 23 de julho de 2015. Aprovado em 18 de janeiro de 2016. 


\section{ANEXOS}

Tabela A1 Estimativas para a fração dos consumidores restritos dados anuais, 1947 - 2012

\begin{tabular}{|c|c|c|c|c|}
\hline Especificação & Instrumentos & Estimativa de $\lambda$ & Desvio padrão & Teste DWH \\
\hline 1 & Nenhum (MQO) & ${ }^{*} 0,94$ & 0,10 & - \\
\hline 2 & $\Delta Y_{t-2}, \ldots, \Delta Y_{t-4}$ & ${ }^{*} 0,93$ & 0,26 & $0,002^{N S}$ \\
\hline 3 & $\Delta Y_{\mathrm{t}-2}, \ldots, \Delta Y_{\mathrm{t}-6}$ & ${ }^{*} 1,13$ & 0,25 & $0,84^{\text {NS }}$ \\
\hline 4 & $\Delta C_{\mathrm{t}-2}, \ldots, \Delta C_{\mathrm{t} \cdot 4}$ & ${ }^{* *} 0,68$ & 0,30 & $0,93^{\text {NS }}$ \\
\hline 5 & $\Delta C_{t-2}, \ldots, \Delta C_{t-6}$ & ${ }^{*} 0,93$ & 0,27 & $0,00^{\text {NS }}$ \\
\hline 6 & $\begin{array}{l}\Delta Y_{\mathrm{t} \cdot 2}, \ldots, \Delta Y_{\mathrm{t}-4} ; \\
\Delta C_{\mathrm{t}-2}, \ldots, \Delta C_{\mathrm{t}-4}\end{array}$ & ${ }^{*} 0,84$ & 0,24 & $0,19^{\text {NS }}$ \\
\hline Média & - & 0,91 & - & - \\
\hline
\end{tabular}

Fonte: Resultados da pesquisa.

Nota: a equação estimada inclui uma constante; * $e^{* *}$ representam significância a $1 \%$ e a $5 \%$, respectivamente; NS denota não significativo.

Tabela A2 Resultados dos testes de raiz unitária ADF e Zivot e Andrews (1992)

\begin{tabular}{|c|c|c|c|c|}
\hline \multicolumn{5}{|l|}{ Painel i: log-consumo } \\
\hline Parâmetros exógenos & Quebras & Defasagens & $t$ calculado & $t$ tabelado \\
\hline Nenhum & Não - ADF & 7 & 0.73 & $-1,95$ \\
\hline Constante & Não - ADF & 8 & $-2,22$ & $-2,91$ \\
\hline Constante e tendência & Não - ADF & 7 & $-1,52$ & $-3,48$ \\
\hline Constante e tendência & $\operatorname{Sim}-(C)$ & 7 & $-3,47$ & $-4,93$ \\
\hline Constante e tendência & $\operatorname{Sim}-(T)$ & 7 & $-4,44$ & $-4,42$ \\
\hline Constante e tendência & $\operatorname{Sim}-(C)$ e $(T)$ & 7 & $-4,32$ & $-5,08$ \\
\hline \multicolumn{5}{|l|}{ Painel ii: log-renda } \\
\hline Parâmetros exógenos & Quebras & Defasagens & $t$ calculado & $t$ tabelado \\
\hline Nenhum & Não - ADF & 2 & 0,53 & $-1,95$ \\
\hline Constante & Não - ADF & 1 & $-2,51$ & $-2,91$ \\
\hline Constante e tendência & Não - ADF & 1 & $-1,04$ & $-3,48$ \\
\hline Constante e tendência & $\operatorname{Sim}-(C)$ & 1 & $-3,02$ & $-4,93$ \\
\hline Constante e tendência & $\operatorname{Sim}-(T)$ & 1 & $-4,31$ & $-4,42$ \\
\hline Constante e tendência & $\operatorname{Sim}-(C)$ e $(T)$ & 1 & $-4,68$ & $-5,08$ \\
\hline
\end{tabular}

Notas: $(C)$ representa quebra na constante e $(T)$ na tendência. Número de defasagens escolhido pelo critério de informação de Akaike (1987). 
Tabela A3 Teste de cointegração sem quebras estruturais

\begin{tabular}{l|r|r|r|r}
\hline Teste ADF & $\begin{array}{r}\text { Coeficiente } \\
\text { estimado }\end{array}$ & Erro padrão & Valor $\boldsymbol{c}$ & $\boldsymbol{p}$-valor \\
\hline Saídas do teste & -0.40 & 0.11 & -3.55 & 0,039 \\
\hline Valor crítico 1\% & & & $-3,73$ & \\
\hline Valor crítico 5\% & & $-3,17$ & \\
\hline Valor crítico 10\% & & $-2,91$ & \\
\hline
\end{tabular}

Fonte: Resultados da pesquisa.

Nota: Foram utilizadas seis defasagens na equação do teste ADF, de acordo com o critério Akaike (1987). P-valor fornecido por MacKinnon (1996).

Tabela A4 Teste de cointegração com quebras estruturais

\begin{tabular}{lr|r}
\hline Teste ADF & Saídas do teste & Data da quebra \\
\hline Estatística calculada & -4.73 & 1985 \\
\hline Valor crítico 1\% & $-5,47$ & \\
\hline Valor crítico $5 \%$ & $-4,95$ & \\
\hline Valor crítico 10\% & $-4,68$ & \\
\hline
\end{tabular}

Fonte: Resultados da pesquisa.

Nota: Os critérios de Akaike (1987) e o bayesiano de informação indicaram zero defasagem. 